\title{
Bases teóricas y metodológicas para el análisis de depósitos de tsunamis en playas arenosas
}

\author{
Carolina Pía Villagrán \\ Programa de Geografía Física Aplicada, Departamento de Ciencias Sociales, Facultad de \\ Ciencias Sociales y Económicas. Universidad de La Serena. La Serena, Chile \\ pia0903@gmail.com \\ José Enrique Novoa Jerez \\ Programa de Geografía Física Aplicada, Departamento de Ciencias Sociales, Facultad de \\ Ciencias Sociales y Económicas. Universidad de La Serena. La Serena, Chile \\ Centro de Estudios Avanzados en Zonas Áridas (CEAZA) \\ jnovoa@userena.cl
}

\begin{abstract}
RESUMEN
Mediante una exhaustiva revisión bibliográfica, se aborda la caracterización de los depósitos de tsunami históricos y de paleotsunamis a nivel mundial en función de la geomorfología de playas, tomándose en consideración los métodos, técnicas y criterios utilizados en su análisis. Tales experiencias demandan de una sistematización y conceptualización que favorezcan dar respuestas a dónde y cómo determinar áreas de depósitos de tsunami pasados para esta unidad morfológica. Este análisis permitió detectar factores determinantes de esta unidad litoral en los procesos de alta energía de los tsunamis, los impactos, además de la importancia de la playa como fuente de investigación de eventos recientes.
\end{abstract}

Palabras Clave: Depósitos de Tsunami, geomorfología litoral, distribución mundial.

\section{Theoretical and methodological bases for analysis of tsunami deposits of sandy beaches}

\begin{abstract}
Through extensive literature review deals with the characterization of historic tsunami deposits and global paleotsunamis depending on the geomorphology of beaches, taking into account the methods, techniques and criteria used in their analysis. Such experiences require a conceptualization that encourage systematization and give answers to where and how to identify areas of past tsunami deposits for this morphological unit. Analysis allowed detection of determinants of the coastal drive in high-energy processes of tsunamis, impacts, and the importance of the beach as a research of recent events.
\end{abstract}

Key words: Tsunami deposits, coastal geomorphology, worldwide distribution. 


\section{INTRODUCCIÓN}

Tsunami se define como ondas de longitud largas generadas por perturbaciones de corta duración y gran extensión en el fondo marino o en su superficie que impulsa y desplaza verticalmente la columna de agua (MCMURTRY et al. 2004, DABRIO y POLO 2005, WEBSTER et al. 2007). Al aproximarse a la costa, las olas generadas descargan su energía pudiendo inundar grandes superficies litorales, siendo capaces de causar alteraciones en la geomorfología y sedimentología de las costas (DAWSON et al. 1991; BRYANT et al. 1996, LAGOS 2000, BRYANT y NOTT 2001, SCHEFFERS y KELLETAT 2003, SINGARASUBRAMANIAN et al. 2006), comprobados en la estratigrafía y vestigios del lugar (PETERS et al. 2007). Estos mecanismos de alteración en la columna de agua, son asociados a causas como sismos, erupciones volcánicas, deslizamientos de tierra submarinos, caída de meteoritos o desprendimiento de hielos (EIPER 2004, FAGHERAZZI y DU 2008, BOURGEOIS 2009). Predominan los tsunamis de origen sísmico en zonas de subducción tectónica, donde es posible encontrar rupturas cercanas al foco (e.g., el margen continental del Océano Pacífico como Chile en América del Sur, Cascadia en América del Norte, y NW de Asia).

Para buscar los mecanismos de liberación de alta energía y las nuevas formas de desequilibrio en los ambientes costeros, en los últimos años se han realizado esfuerzos para detallar y generar inventarios de antecedentes e investigaciones sobre tsunamis pasados en las costas del mundo (NOVOA 1995, RUEGG \& RUDLOFF 2009). Los devastadores maremotos recientes se han constituido en laboratorios naturales de los procesos hidrodinámicos y sedimentarios para dilucidar la característica cíclica de estos fenómenos (Perú 2001, Sumatra 2004 y Chile 2010). Involucran procesos de erosión y depositación que dejan evidencias distintivas en cada unidad que conforma el ambiente litoral (playa, duna, plataforma litoral, humedal), razón que sustenta la presente recopilación de antecedentes a fin de proponer un enfoque geomorfológico, el que ha sido escasamente utilizado, centrando consecuentemente el análisis en la unidad de playa y sus respectivos procesos litorales para facilitar posteriores investigaciones que consideren los depósitos de tsunami.

\section{MATERIALES Y MÉTODOS}

Los primeros trabajos sobre interpretación de depósitos de tsunami fueron descritos para la isla hawaiana de Lanai (MOORE \& MOORE 1984), iniciándose una amplia labor investigativa (PÉREZ-TORRADO et al. 2002) en las costas de Washington (ATWATER 1987), Escocia (DAWSON et al. 1991) y Chile (PASKOFF 1991). La década de los noventa marca el inicio de una sucesión de importantes publicaciones en relación al tema (BRYANT et al. 1992; NISHIMURA \& MIYAJI 1995; SYNOLAKIS 1997; MINOURA et al. 2001; SCHEFFERS 2002; WHELAN \& KELLETAT 2002; SCHEFFERS 2004; FUJINO et al. 2006; BAHLBURG \& WEISS 2007; GOTO et al. 2007; FAGHERAZZI \& DU 2008, SUZUKI et al. 2008; GOTO et al. 2009; PIGNATELLI et al. 2009).

Es posible distinguir las investigaciones sobre paleotsunamis que corresponden a evidencias geomorfológicas y geológicas anteriores a los registros históricos (PASKOFF 1991; DAWSON 1999; DAWSON \& SHI 2000; BRYANT \& NOTT 2001; HARTLEY et al. 2001; MINOURA et al. 2001; PINEGINA \& BOURGEOIS 2001; BRYANT \& HASLETT 2002; SCHEFFERS 2002; WHELAN \& KELLETAT 2002; SCHEFFERS \& KELLETAT 2003; YAMANO et al. 2003; MCMURTRY et al. 2004; SCHEFFERS 2004; SCHEFFERS \& KELLETAT 2004; CANTALAMESSA \& DI CELMA 2005; LE ROUX \& VARGAS 2005; FUJINO et al. 2006; MORTON et al. 2007; DAWSON \& STEWART 2007; KORTE- 
KAAS \& DAWSON 2007; MCMURTRY et al. 2007; PETERS et al. 2007; PUGABERNABÉU et al. 2007; SCHEFFERS \& SCHEFFERS 2007; KELLETAT 2008; LE ROUX et al. 2008; SCHEFFERS et al. 2008; MAOUCHE et al. 2009; GOFF et al. 2010a; GOFF et al. 2010b) de los tsunamis históricos con evidencias geográficas, escritas e incluso orales, destacándose el amplio registro existente en Japón y antecedentes post evento de Sumatra el 26 de diciembre del 2004 (LAGOS 2000; MATSUMOTO et al. 2001; JAFFE et al. 2003; MASTRONUZZI \& SANZO 2004; NARAYAN et al. 2005; KIRCA \& KABDASLÝ 2006; RICHMOND et al. 2006; SZCZUCINSKI et al. 2006; BAHLBURG \& WEISS 2007; GOTO et al. 2007; NANAYAMA et al. 2007; CHOOWONG et al. 2008; FAGHERAZZI \& DU 2008; IMAMURA et al. 2008; SUZUKI et al. 2008; HART \& KNIGHT 2009; HULUGALLE et al. 2009; PARIS et al. 2009; SANTOS et al. 2009; SRINIVASALU et al. 2009; SUGAWARA et al. 2009; GOTO et al. 2010a; ILAYARAJA \& KRISHNAMURTHY 2010; PARIS et al. 2010; SRISUTAM \& WAGNER
2010). Para establecer la diferencia entre ambos conceptos temporales de tsunamis, se requiere de mecanismos de datación relativa y absoluta para establecer la certeza morfogenética del depósito, sustentada en las evidencias sedimentarias y geomorfológicas para alcanzar una objetiva comprensión del fenómeno. En muchos casos, las investigaciones de registros sedimentarios de tsunamis históricos han aumentado el registro más allá de los antecedentes existentes, identificando fenómenos más antiguos, hasta paleotsunamis en litorales con escaso a nulo registro temporal de estos eventos.

La figura 1 señala la distribución espacial de las investigaciones de depósitos de tsunami en el mundo. Si se compara con los mapas de SCHEFFERS \& KELLETAT (2003), LAGOS \& CISTERNAS (2004) y GOTO et al. (2010a), se distingue un creciente impulso en estos temas en distintas costas del mundo. Por otro lado, si la escala permitiese visualizar en un mayor detalle, se daría a conocer que el continuo aumento de investigaciones presenta un patrón espacial concentrado en las áreas más investigadas

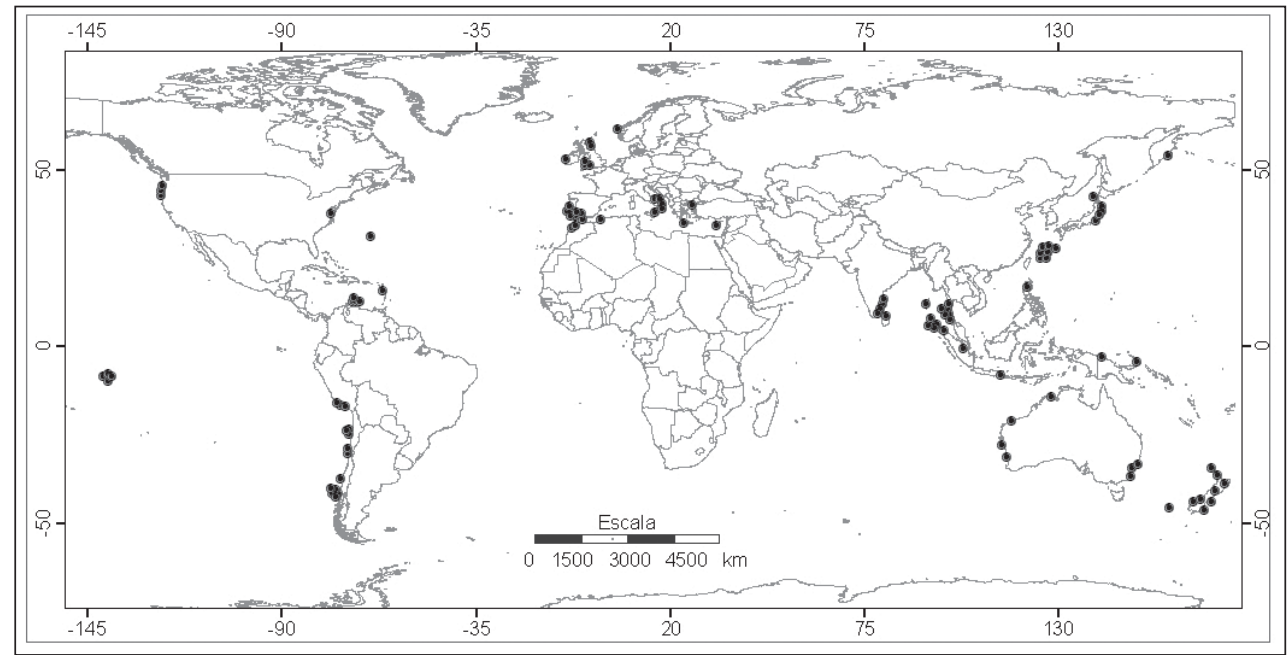

Fig. 1. Espacialización de investigaciones de depósitos de tsunami en el mundo. Elaboración propia en base a tabla 1.

Fig 1. Spatialization research of tsunami deposits in the world. Prepared by authors based on Table 1. 
(Tailandia, India, Estados Unidos, Japón, España, Portugal, Nueva Zelanda, Australia y la costa de América del Sur con Chile y Perú). Situación que se produce por nuevos eventos y depósitos en áreas ya estudiadas, enriquecimiento con nuevos métodos de los resultados existentes, mayor cantidad de datos de campo y, en caso inverso, por la dificultad de encontrar nuevos depósitos en otras áreas. Esta situación hace necesario emprender investigaciones de este tipo en las costas sin antecedentes, además de valorar los métodos y criterios ya establecidos.

En los últimos años, los tsunamis y sus registros sedimentarios en el mundo, han sido objeto de estudio por diversos investigadores, aunque persiste una escasez de ellos debido a lo reciente de esta temática (SCHEFFERS \& KELLETAT 2003), otorgándole relevancia científica a esta línea de investigación, valoración e incentivo a la comprensión de los procesos propios de la geomorfología litoral ligados a fenómenos de alta energía como los tsunamis, situación que favorece la elaboración de un soporte conceptual para nuevos hallazgos de depósitos de tsunami en lugares poco estudiados o potencialmente analizables en el futuro.

\section{Criterios de análisis de depósitos de tsunami}

Se distingue la sorprendente energía liberada en procesos de tsunami, plasmando su poder erosivo y depositacional, al dejar como resultado huellas y sedimentos en las zonas litorales del mundo. Estos registros, presentan distinta forma, tamaño y textura, en directa relación con la naturaleza del lugar donde se encuentren, aspecto relevante para el análisis en trabajos relacionados con depósitos de tsunami cuyo criterio básico es la distinción del tamaño del sedimento a detallar.

Numerosos documentos explican las depositaciones de tsunamis, que basados en la identificación de capas de sedimento alóctono en la estratigrafía costera, centran sus análisis en el tamaño de los sedimentos encontrados: finos (DAWSON 1999; DAWSON \& SHI 2000; MINOURA et al. 2001, SCHEFFERS 2002; ANDRADE et al. 2003; BONDEVICK et al. 2005; NANAYAMA et al. 2007; HULUGALLE et al. 2009; OLIVEIRA et al. 2009; SRINIVASALU et al. 2009; SRISUTAM \& WAGNER 2010), gruesos (NOTT 2000; BRYANT \& NOTT 2001; BRYANT \& HASLETT 2002, FELTON \& CROOK 2003, MCMURTRY et al. 2004; SCHEFFERS \& KELLETAT 2004; NISHIMURA et al. 2005; MORTON et al. 2006; BRYANT \& HASLETT 2007; MASTRONUZZI et al. 2007; WEBSTER et al. 2007; MAOUCHE et al. 2009; GOTO et al. 2010a) y de enormes dimensiones denominados Megaboulders (PASKOFF 1991; KELLETAT et al. 2007; MASTRONUZZI et al. 2007; MHAMDI \& MEDINA 2008; SCHEFFERS et al. 2008; PARIS et al. 2009). Además, se encuentra la clasificación de depósitos bimodales que corresponden a mezclas de arenas y cantos rodados o mezcla bimodal, depósito característico de tsunami que presenta una estructura caótica, donde puede dominar la arena o bien las rocas y cuya variabilidad depende de las condiciones locales en donde se encuentre (KEATING et al. 2004; SCHEFFERS \& KELLETAT 2004; KELLETAT et al. 2007).

Otro criterio es la comparación energética que se destaca desde los inicios de esta línea de investigación, la importancia de establecer diferencias entre los depósitos de alta energía (tsunami) y otros eventos (tormentas, ciclones tropicales, tifones) para corroborar la validez del depósito como de origen tsunámico (Australia, Portugal, Hawai, Reino Unido, Italia, Japón, entre otros). La tarea de analizar y detectar diferencias entre depósitos de tsunamis y tormentas ha sido una dificultosa búsqueda de criterios, cuya escasa información inicial limitaba las diferenciaciones al no presentar características definitivas. Los progresivos estudios de estos aspectos contrastantes permitió presentar factores diferenciales con mayor validez que se han mantenido como constante en el 
tiempo (DAWSON \& SHI 2000; BRYANT \& NOTT 2001; FELTON \& CROOK 2003; YAMANO et al. 2003; GOFF et al. 2004; MASTRONUZZI \& SANSO 2004; NOTT 2004; SCHEFFERS 2004; WILLIAMS \& HALL 2004; CISTERNAS 2005; GOFF et al. 2006; BAHLBURG \& WEISS 2007; BRYANT \& HASLETT 2007; FUJIWARA \& TAMATAKI 2007; KORTEKAAS \& DAWSON 2007; MORTON et al. 2007; NANAYAMA et al. 2007; KELLETAT 2008; SPISKE et al. 2008; SUZUKI et al. 2008; GOFF et al. 2009; GOTO et al. 2009; ENGEL et al. 2010; GOTO et al. 2010a; GOTO et al. 2010b; MITCHELL et al. 2010). Los análisis de contrastes entre tsunami y tormentas, se diferencian por la caracterización morfológica, morfodinámica y morfogenética de los depósitos (Tabla 1).

Entre los métodos utilizados están las ecuaciones hidrodinámicas de NOTT (1997 y 2002) que relacionan la fuerza requerida por la onda para el transporte de rocas en zonas costeras, aspectos litológicos (tamaño, posición y orientación), utilización de fotos aéreas o material gráfico, discriminación de rocas de grandes dimensiones y peso para descartar la acción de las olas por tormentas, análisis de conjuntos de datos y evaluación de depósitos para ambos eventos en una misma área, zanjas para análisis estratigráfico, esquemas o bosquejos de diferencias y comparación estadística de los resultados antes mencionados. El esclarecimiento de la génesis de estos dos tipos de depósitos se fundamenta por diferencias en su hidrodinámica y la paleontología, donde la periodicidad de la llegada de los trenes de ola condicionaría la diferenciación estratigráfica entre los depósitos de tsunami y tormenta. Por lo tanto, el detectar el origen del depósito se ve facilitado por la sedimentación resultante, el análisis de su estratigrafía y paleontología, además de la corroboración de datos históricos bien detallados para ambos eventos.

Se puede rescatar que los depósitos de tsunami generalmente se presentan en capas de poco espesor $(<50 \mathrm{~cm})$ con un rango recurrente de 5 a $25 \mathrm{~cm}$ que puede contener una lámina individual sin estructura o una formación de varias capas delgadas. Además, tienden a poseer láminas de barro en su interior, presencia de metales pesados, foraminíferos y escasa organización en su estructura, presenta sectores gruesos pero tienden a adelgazarse hacia el continente y una gran extensión (hasta kilómetros) hacia el interior. Mientras que los depósitos de tormenta, contienen numerosas capas laminares cuyos rangos de espesor van de 25 a $200 \mathrm{~cm}$ y suelen poseer fragmentos de conchas y otros elementos, pero presenta en su estructura mayor organización, menor alcance y poder de transporte. Estas diferencias tienen directa relación con la dinámica de estos eventos, FUJIWARA \& KAMATAKI (2007) estiman que la longitud de onda del tsunami es 100 veces más larga que la longitud de ondas de tormenta. 
TABla i. Clasificación de las investigaciones de depósitos de tSUNAMi En PLAYa. TABLE I. RESEARCH OF TSUNAMI DEPOSITS ON THE BEACH CLASSIFICATION.

\begin{tabular}{|c|c|}
\hline $\begin{array}{c}\text { PROCESOS } \\
\text { EXPLICATIVOS }\end{array}$ & Playa \\
\hline MORFOLOGÍA & $\begin{array}{l}\text { DAWSON \& SHI (2000), NOTT (2000), LUQUE et al.(2001), MINOURA et al. (2001), } \\
\text { JAFFE \& GELFENBAUM (2002), SCHEFFERS (2002), WHELAN \& KELLETAT (2002), } \\
\text { JAFFE et al. (2003), SCHEFFERS \& KELLETAT (2003), EIPER (2004), KEATING et } \\
\text { al. (2004), KELLETAT \& SCHEFFERS (2004), MASTRONUZZI \& SANSO (2004), MC- } \\
\text { MURTRY et al. (2004), SCHEFFERS (2004), WILLIAMS \& HALL (2004), CANTALA- } \\
\text { MESSA \& DI CELMA (2005), LE ROUX \& VARGAS (2005), FUJINO et al. (2006), } \\
\text { RICHMOND et al. (2006), SINGARASUBRAMANIAN et al. (2006), SZCZUCINSKI et } \\
\text { al. (2006), BAHLBURG \& WEISS (2007), DOMINEY-HOWES (2007), FUJIWARA \& } \\
\text { TAMATAKI (2007), GOTO et al. (2007), KORTEKAAS \& DAWSON (2007), PETERS } \\
\text { et al. (2007), MASTRONUZZI et al. (2007), MCFADGEN \& GOFF (2007), MCMUR- } \\
\text { TRY et al. (2007), MOORE \& JAFFE (2007), MORTON et al. (2007), SCHEFFERS } \\
\text { \& SCHEFFERS (2007), FAGHERAZZI \& DU (2008), LE ROUX (2008), LUQUE et al. } \\
\text { (2008), MHAMMDI et al. (2008), SUZUKI et al. (2008), BOURGEOIS (2009), FUJINO } \\
\text { et al. (2009), HULUGALLE et al. (2009), PARIS et al. (2009), NICHOL et al. (2010), } \\
\text { SRISUTAM \& WAGNER (2010). }\end{array}$ \\
\hline MORFOGÉNESIS & $\begin{array}{l}\text { DAWSON \& SHI (2000), NOTT (2000), LUQUE et al. (2001), MINOURA et al. (2001), } \\
\text { SCHEFFERS \& KELLETAT (2003), EIPER (2004), KIRCA \& KABDASLI (2004), KEA- } \\
\text { TING et al. (2004), LAGOS \& CISTERNAS (2004), MASTRONUZZI \& SANSO (2004), } \\
\text { MCMURTRY et al. (2004), SCHEFFERS (2004), CANTALAMESSA \& DI CELMA } \\
\text { (2005), FUJINO et al. (2006), RICHMOND et al. (2006), SZCZUCINSKI et al. (2006), } \\
\text { BAHLBURG \& WEISS (2007), DAWSON \& STEWART (2007), DOMINEY-HOWES } \\
\text { (2007), FUJIWARA \& TAMATAKI (2007), MASTRONUZZI et al. (2007), MCFADGEN } \\
\text { \& GOFF (2007), MCMURTRY et al. (2007), MORTON et al. (2007), PETERS et al. } \\
\text { (2007), FAGHERAZZI \& DU (2008), LE ROUX et al. (2008), BOURGEOIS (2009), } \\
\text { FUJINO et al. (2009), ARAOKA et al. (2010), GOTO et al. (2010b), ILAYARAJA \& KRIS- } \\
\text { HNAMURTHY (2010), MAOUCHE et al. (2010). }\end{array}$ \\
\hline MORFODINÁMICA & $\begin{array}{l}\text { DAWSON (1999), DAWSON \& SHI (2000), NOTT (2000), LUQUE et al. (2001), MI- } \\
\text { NOURA et al. (2001), JAFFE \& GELFENBAUM (2002), SCHEFFERS (2002), WHE- } \\
\text { LAN \& KELLETAT (2002), JAFFE et al. (2003), SCHEFFERS \& KELLETAT (2003), } \\
\text { GOFF et al. (2004), KEATING et al. (2004), KELLETAT \& SCHEFFERS (2004), KIR- } \\
\text { CA \& KABDASLI (2004), LAGOS \& CISTERNAS (2004), MASTRONUZZI \& SANSO } \\
\text { (2004), MCMURTRY et al. (2004), SCHEFFERS (2004), SCHEFFERS \& KELLETAT } \\
\text { (2004), WILLIAMS \& HALL (2004), CANTALAMESSA \& DI CELMA (2005), LAGOS \& } \\
\text { GUTIÉRREZ (2005), LE ROUX \& VARGAS (2005), FUJINO et al. (2006), GOFF et } \\
\text { al. (2006), RICHMOND et al. (2006), SINGARASUBRAMANIAN et al. (2006), SZCZU- } \\
\text { CINSKI et al. (2006), BAHLBURG \& WEISS (2007), DAWSON \& STEWART (2007), } \\
\text { DOMINEY-HOWES (2007), FUJIWARA \& TAMATAKI (2007), GOTO et al. (2007), } \\
\text { HASLETT \& BRYANT (2007), KORTEKAAS \& DAWSON (2007), MASTRONUZZI et } \\
\text { al. (2007), MCFADGEN \& GOFF (2007), MCMURTRY et al. (2007), MOORE \& JA- } \\
\text { FFE (2007), MORTON et al. (2007), NANAYAMA et al. (2007), PETERS et al. (2007), } \\
\text { SCHEFFERS \& SCHEFFERS (2007), VÖTT et al. (2007), WEBSTER et al. (2007), } \\
\text { CHOOWONG et al. (2008), FAGHERAZZI \& DU (2008), IMAMURA et al. (2008), LU- } \\
\text { QUE et al. (2008), MHAMMDI et al. (2008), PRITCHARD \& DICKINSON (2008), SU- } \\
\text { ZUKI et al. (2008), BOURGEOIS (2009), FUJINO et al. (2009), GOTO et al. (2009), } \\
\text { HULUGALLE et al. (2009), MAOUCHE et al. (2009), PARIS et al. (2009), PIGNATELLI } \\
\text { et al. (2009), SANTOS et al. (2009), SUGAWARA et al. (2009), SRINIVASALU et al. } \\
\text { (2009), ARAOKA et al. (2010), GOTO et al. (2010),GOTO et al. (2010b), ILAYARAJA } \\
\text { \& KRISHNAMURTHY (2010), NICHOL et al. (2010), PARIS et al. (2010), SRISUTAM } \\
\text { \& WAGNER (2010). }\end{array}$ \\
\hline
\end{tabular}




\section{RESULTADOS}

\section{Tsunamis y Análisis geomorfológico en playa}

La revisión realizada permite comprobar la capacidad de los tsunamis para dejar evidencias y modificar formas en el litoral. Las ondas a su paso pueden erosionar, transportar sedimentos y depositar gran volumen de material en poco tiempo a cientos de metros y hasta kilómetros de distancia de la costa. Ésto hace que dentro de los diversos enfoques, existan contribuciones que se acercan al rol de la geomorfología para facilitar la identificación de vestigios y depósitos de tsunamis pasados, pero éstas son descripciones que integran procesos y formas que consideran el punto de vista hidrológico, sedimentario, y morfodinámico, entre otros. En este documento se analizan estas contribuciones para la unidad morfológica de playa. Se hace la diferencia en la caracterización morfológica, morfodinámica y explicación de la génesis de los depósitos y en cada proceso se detalla una síntesis de las descripciones revisadas.

\section{Morfología}

Se identificó depósitos y huellas de tsunami en playas de varias costas del mundo pudiéndose distinguir entre costas arenosas y rocosas (Japón, Indonesia, Tailandia, Hawaii, Nueva Zelanda e Italia, entre otras), constituyéndose en la unidad morfológica más estudiada. Varios autores plantean la dificultad en la discriminación entre los depósitos de tsunami respecto de los depósitos asociados a fenómenos con mayor recurrencia y constante acción morfodinámica (tormentas, bravezas, ciclones).

La configuración costera y las características de la plataforma representan factores importantes en la llegada del oleaje a la costa, debido a que en costas escarpadas con costas refletivas y con abruptos cambios en la plataforma, el oleaje erosiona el acantilado en condiciones regulares que en condiciones de tsunami ve incrementada progresivamente las velocidades de onda, aumentando la capacidad de transporte de los clastos en el litoral. En costas deposicionales como las playas arenosas, se distingue la movilidad del material transportado por el lavado de retroceso que ayuda a la formación de depósitos sedimentarios estratificados, así como modificaciones en superficie por los procesos de erosión en el perfil de playa, en las bermas y dunas presentes. La vegetación herbácea es analizada por la ruptura de sus tallos e inclinación conforme al flujo, así como por la presencia de acumulación de restos vegetales y microfósiles. En estos análisis destaca la particularidad morfológica costera asociada a la presencia de acantilados, barreras naturales y artificiales que influyen en la magnitud de la erosión y en el impacto del tsunami (las islas barrera, islas y remanentes rocosos, rompeolas, vías de comunicación y edificaciones, cuyo obstáculo genera un efecto amortiguador del impacto en las costas y tierra adentro).

Los métodos utilizados en las investigaciones de costas arenosas, principalmente en sondeos posteriores a tsunamis recientes, se sustentan en la identificación de rocas y bloques de carácter alóctono cuya procedencia y evidencias ratifiquen el transporte de tsuna$\mathrm{mi}$, capas de sedimentos que consideran su localización y estructura interna (excavaciones de zanjas, seguimiento de transectos, extracción de testigos), análisis de campo, observaciones de primera fuente (espectadores y fotografías del suceso), mediciones del flujo para establecer altura de escorrentía y runup (troncos inclinados, pilares y escombros, marcas de nivel de inundación), informaciones obtenidas por sistemas de comparación remota y cercana de áreas afectadas pre y post evento (teledetección, material gráfico y videos). Por otro lado, para el estudio de los eventos de carácter histórico y paleotsunamis, se distingue la complementación de información con registros arqueológicos y bioestratigráficos.

En las costas rocosas generalmente se producen desprendimientos de grandes rocas, los métodos utilizados destacan el uso de 
teorías y modelos hidrodinámicos que calculan la magnitud mínima capaz de mover las rocas y depósitos hacia el continente para evaluar los cantos dispersos en la costa. Otros métodos y técnicas consideran la presencia de fósiles, biota marina y corales, la orientación y localización de los bloques, en contraste con los cálculos hidrodinámicos del proceso. Complementariamente a los métodos mencionados, suelen usarse técnicas de datación que permiten estimar intervalos de recurrencia mediante radiocarbono, Cesium 137, dendrocronología de los árboles y OSL (Optically Stimulated Luminescence) para optimizar su exactitud.

\section{Morfogénesis}

Los depósitos de tsunami en playa, se distinguen por procesos relacionados con tsunamis generados por fuertes sismos, desprendimiento de flancos de un volcán submarino e impacto de meteoritos en épocas prehistóricas. Los fenómenos desencadenantes corresponden a eventos cercanos y lejanos. La elevada cantidad de registros en variados tipos de costas, muestra a esta unidad morfológica como un ambiente idóneo para estudios post-evento de carácter reciente. En el caso de paleotsunamis, se dificulta la investigación en playa debido a la vulnerabilidad de estas formas a ser sometidas a removilización y reelaboración de depósitos por eventos de alta energía recurrentes y no recurrentes a través del tiempo. De manera que cuando se dispone de un depósito o huella anómalas en estas unidades morfológicas, es preciso establecer diferenciaciones entre los depósitos de tsunami con aquellos periódicos (tormentas, mareas vivas y ciclones) ya que la causante del transporte de los sedimentos debe coincidir con depositaciones de alta energía para descartar otra fuente de generación.

\section{Morfodinámica}

En playas y bahías se disipa la energía que acompaña a la masa de agua que viaja desde el mar de fondo a la plataforma y descar- ga en la costa, reduciéndose la longitud de onda e incrementándose la altura de la onda de tsunami por el efecto de fricción de fondo. El resultado se refleja en una serie de trenes de olas para cuya dinámica es fundamental la configuración de la costa. Si la costa presenta una suave pendiente, la energía del tsunami se proyectará a cabalidad. En el caso de bahías que poseen macizos rocosos y penínsulas, puede haber reflexión en los bordes de las costas y, con ello, aumenta el tiempo de la onda en recorrer la bahía. No obstante, un segundo tren de ola puede verse reforzado con un remanente del primer tren y aumentar la energía al interior de la bahía (fenómeno conocido como resonancia), situación que puede aumentar la altura de las olas, alterando la condición inicial de la altura de la onda que ingresa en tierra (run-up), al igual que su poder erosivo, efectos en la sedimentación y nivel de inundación. De manera que dentro de los antecedentes revisados es importante estimar la altura que alcanza la ola para incidir en las costas deposicionales y rocosas. Se debe considerar también que la altura puede ser afectada por el ancho de la plataforma continental, batimetría costera, alteraciones en la topografía submarina, rugosidad y formas del litoral, ya sea en bahías cerradas o costas abiertas. Este proceso morfodinámico da como resultado una sedimentación que se desprende de los mecanismos de ingreso y flujos de retorno (cuyos efectos se registran en capas de sedimentación, depositación de rocas y bloques de gran tamaño provenientes del supra y sublitoral), pueden dejar muestras de erosión en la playa (en bermas, áreas rocosas aledañas, campos de dunas, evidencias en la vegetación), modificando el balance sedimentario regular de la playa, quedando plasmado en la morfología y estratigrafía del lugar.

La ubicación de las investigaciones en la unidad de playa (Fig. 2) presenta una distribución similar a la de los estudios de depósitos en el mundo en general (Fig. 1). La concentración de los estudios en ciertas costas del mundo, presentan numerosas contri- 


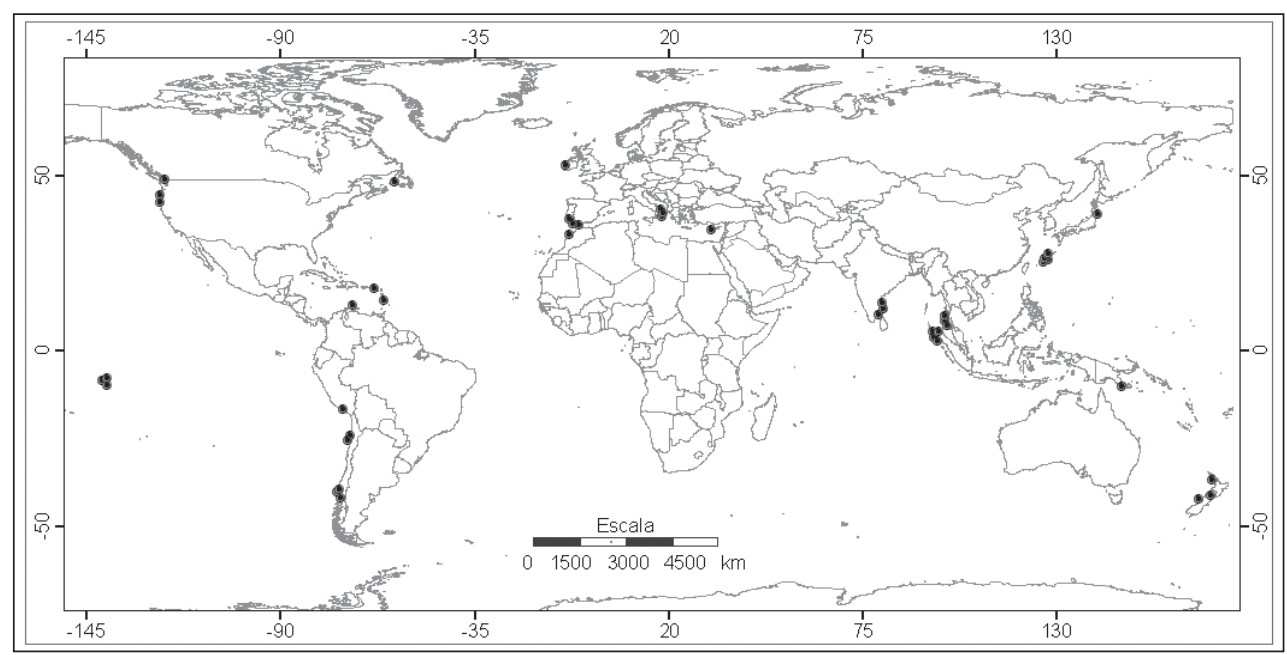

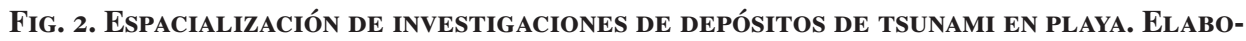
RACIÓN PROPIA EN BASE A TABLE $I$.

Fig. 2. Spatialization research of tsunami deposits on the beach. Prepared by authors based ON TABLE 1.

buciones y avances en la materia, siendo la playa una unidad de análisis fundamental para los depósitos y huellas de tsunamis debido a la alta morfodinámica presentada en estos eventos y la modificación del paisaje en tsunamis recientes.

\section{DISCUSIÓN}

\section{Métodos de análisis de discriminación}

La revisión de los distintos análisis de depósitos de tsunami que inciden la morfología de playa, permite distinguir que en sus metodologías de trabajo consideran fuentes de información de diverso origen (antecedentes orales, interpretaciones de material visual, análisis de campo y modelaciones basadas en cálculos matemáticos, entre otros), con el fin de optimizar la información disponible y extraíble del fenómeno, considerando de esta manera todas las contribuciones posibles para su investigación, generándose un cruce de referencias primarias y secundarias que añaden valor a la comprensión analítica de estos fenómenos naturales. Aspecto que ha sido motivo de cuestionamientos, debido a la distinta naturaleza de cada fuente de información, pero que sin embargo es considerada útil y válida para el desarrollo de metodologías y la obtención de resultados que conjuntamente se relacionan con datos cronológicos para su precisión (WHELAN \& KELLETAT 2002; SINGARASUBRAMANIAN et al. 2006; MCFADGEN \& GOFF 2007; GOTO et al. 2010b). Esta complejidad de análisis se gestaría debido a que estos eventos de alta energía involucran varios procesos en su incidencia en la costa, alterando el comportamiento geomorfológico y sedimentológico en unidades morfológicas costeras como es el caso de la playa, siendo valorable toda información extraíble de estas alteraciones post evento.

$\mathrm{Al}$ analizar la morfología costera de playa, se encontraron controversias referidas a la existencia de registros de paleotsunami, como también complejidades en la datación de los depósitos. Mientras más antiguos, 
predominan estimaciones relativas por sobre las absolutas mediante mecanismos de validación que se complementan con antecedentes indirectos. Estas dificultades se deben al carácter cíclico de los eventos cuyos depósitos pueden estar sujetos a removilización y a los efectos de la morfodinámica litoral (problemas en las técnicas de datación, la presencia de eventos diurnos y nocturnos, cantidad insuficiente de materia orgánica e incidencias astronómicas) (DAWSON \& SHI 2000; JAFFE \& GELFENBAUM 2002; MCMURTRY et al. 2004; SCHEFFERS 2004; MASTRONUZZI et al. 2007; PETERS et al. 2007; LE ROUX et al. 2008; SCHEFFERS et al. 2008; SUSUKI et al. 2008; GOFF et al. 2010b).

Aunque los continuos avances tecnológicos han favorecido los mecanismos de datación, la generalidad de los autores no discute el uso de una técnica por sobre otra para mejorar los resultados alcanzados, prefiriéndose utilizar métodos complementarios cuando los recursos económicos de los proyectos lo permiten. De lo anterior se desprende que las técnicas más apropiadas y su efectividad, dependerán de las características del depósito (presencia de materia orgánica, fósiles, foraminíferos, metales pesados, antigüedad relativa) y del nivel de precisión que se pretende lograr. La datación por radiocarbono es aconsejable para depósitos que posean materia orgánica y se busque una menor precisión absoluta, requiriéndose su complementación con métodos hidrodinámicos para comprender su morfodinámica depositacional y discriminar su origen respecto de fenómenos atmosféricooceánicos recurrentes (JAFFE et al. 2002; SCHEFFERS 2002; WHELAN \& KELLETAT 2002; KELLETAT et al. 2007; MASTRONUZZI et al. 2007; SCHEFFERS et al. 2008; GOFF et al. 2009: SUGAWARA et al. 2009; GOTO et al. 2010). La datación mediante OSL se aconseja para establecer con mayor precisión la fecha de un evento y es utilizada para encontrar procesos de recurrencia estadística, asociados con los parámetros geológicos de ruptura que le dieron origen, donde no existe un historial reconocido de eventos pasados (EIPER et al. 2004; KORTEKAAS \& DAWSON 2007). El Cesium 137, técnica que establece de manera muy certera mediciones de la pérdida de suelo producto de la erosión y la redepositación de partículas en el paisaje, permite distinguir los depósitos de tsunamis históricos de los paleotsunamis, importante para la determinación de aspectos cíclicos de estos eventos en costas sísmicamente activas (PETERS et al. 2007). Los métodos dendrocronológicos se recomiendan en costas que posean tectónica activa para discriminar movimientos sísmicos y cosísmicos de solevantamiento y hundimiento en ambientes que posean coberturas vegetacionales apropiadas para su aplicación (WHELAN \& KELLETAT 2002; PETERS et al. 2007).

En playa se complementan diversas técnicas para discriminar depósitos de tsunami respecto de los de tormenta (DAWSON \& SHI 2000; FELTON \& CROOK 2003; KORTEKAAS \& DAWSON 2007; MORTON et al. 2007; SPISKE et al. 2008; GOTO et al. 2009; GOFF et al. 2010a; MITCHELL et al. 2010). Como criterio relevante en la búsqueda de depósitos de tsunami es la identificación de megaclastos en áreas cercanas a la costa (depositados por olas de gran potencial energético) que se apoya en cálculos hidrodinámicos para constatar esa capacidad de carga. No obstante, persisten dificultades cuando la considerable energía desencadenada por fenómenos atmosféricos permiten desplazar sedimentos de proporciones mayores en unidades como la playa, sobrepasándose la norma del "Rule of Thumb” (LOWE \& DE LANGE 2000) ya que en Japón existen evidencias de olas generadas por tifones que han sido capaces de movilizar bloques de hasta 7 toneladas. Problemática que se intenta resolver centrando el análisis del período de las ondas, más que en las alturas de olas de tsunami, su desplazamiento, características (porosidad, densidad, forma y color), contenido de fósiles y distribución de rocas en la costa. 
Para el caso de los depósitos finos en capas de arena en playas o sectores cercanos a la costa, la diferenciación de sedimentos entre estos fenómenos también resulta compleja, basándose en el análisis estratigráfico, distribución espacial, organización, clasificación, presencia de microfósiles y correspondencia cronológica mediante datación.

\section{Métodos de análisis geomorfológico}

Existen contribuciones que intentan este acercamiento geomorfológico para distinguir depósitos y huellas de tsunamis, vinculando procesos con unidades morfológicas en sitios específicos (BRYANT \& NOTT 200; WHELAN \& KELLETAT 2002; SCHEFFERS \& KELLETAT 2003; MORTON et al. 2006; DOMINEYHOWES 2007; LUQUE 2008; GOFF et al. 2009; MAOUCHE et al. 2009; PARIS et al. 2009). No obstante, los estudios no comparan los efectos en mismas morfologías de diferentes costas, sino que en morfologías de un mismo lugar, situación que puede gestarse debido al carácter reciente de estos enfoques.

Se establecen diferenciaciones y aspectos coincidentes en los efectos de tsunami en formas particulares. Sin embargo, el depósito y huella en cada una de las unidades morfológicas está asociado a distintas secuencias o etapas de la morfodinámica de un tsunami, ya sea en su etapa erosiva, de transporte y finalmente en la descarga de energía. En la unidad de playa se puede distinguir la predominancia de procesos erosivos con materiales fácilmente removilizables durante eventos de alta energía como los tsunamis, aspecto que no resulta ser tan determinante ni simple, si se considera el carácter dinámico de la morfología en su evolución. En este sentido, la estabilidad tiene relación con la capacidad de una forma para alcanzar equilibrio en cualquier estado evolutivo (CHURCH 2010), y los tsunami generan desequilibrios en su comportamiento morfodinámico, produciéndose una mayor complejidad al momento de dilucidar los fenómenos morfogenéticos (NANAYAMA et al. 2007). Dificultad que no escapa al planteamiento propuesto para dilucidar mecanismos de paleotsunamis en costas que poseen tectónica activa, determinar el grado de inundación en playas de baja pendiente con material continuamente sujeto a removilización ya sea por tsunamis o eventos recurrentes y depositaciones tsunámicas en costas con desembocaduras de paleocauces.

\section{CONCLUSIONES}

Es fundamental resaltar la importancia de los distintos enfoques y métodos utilizados para el análisis de depósitos de tsunami y paleo-tsunami en el avance del conocimiento sobre esta materia que complementados y relacionados entre sí, permiten constituir patrones en la estratigrafía del depósito, clasificación granulométrica y su complementación con los modelos hidrodinámicos. Por otro lado, uno de los aspectos de mayor inquietud en este ámbito es la discriminación entre depósitos de tsunami y de tormenta en unidades más expuestas como es el caso de las playas, además de la importancia de esta unidad morfológica desde el punto de vista de la planificación territorial del borde costero y el requerimiento de información certera acerca de los límites de inundaciones pasadas y los eventos que se ven implicados en ella.

En el análisis de depósitos y huellas de tsunami en playa, se destacan importantes factores, tales como configuración de la costa ya sea en bahías cerradas o costas abiertas, plataforma continental, batimetría, alteraciones en la topografía submarina, rugosidad y presencia de vegetación en las relaciones con modelos hidrodinámicos. $\mathrm{La}$ utilización de datos de campo (mediciones topográficas, erosión en playa, evidencias en la orientación de la vegetación), la validación con diversas fuentes primarias y secundarias (material gráfico, sondeos, documentación) y para los fenómenos más 
antiguos, su complementación con registros arqueológicos y bioestratigráficos permiten calibrar las estimaciones mediante técnicas de datación basadas en radiocarbono y OSL.

La unidad de playa es considerada un laboratorio natural ideal para investigaciones de depósitos de tsunami reciente, más que para los paleotsunamis. Su elevada morfodinámica genera cambios y pérdida de los depósitos en costas arenosas y rocosas, mostrando complejidad a la hora de disponer de vestigios que puedan tener alto grado de preservación. Ante los antecedentes planteados y discutidos, toda interpretación geomorfológica de los tsunamis requiere considerar la compleja morfodinámica de los fenómenos modeladores del paisaje costero de acuerdo con sus condiciones paleomorfológicas y morfogenéticas.

Como consideración final, el tratamiento de las diversas fuentes de información que tratan las huellas y depositaciones de tsunamis que involucran procesos e interacción en la unidad de playa, se materializan en otorgar una síntesis de puntos concordantes en la materia. Para una indagación detallada en la unidad morfológica de playa, se puede considerar la sistematización presentada en las tablas correspondientes de este documento, donde se entregan antecedentes actualizados de depósitos de tsunami en el mundo.

\section{Agradecimientos}

Por el envío de antecedentes científicos para este documento, se agradece a los doctores Daisuke Araoka (Graduate School of Frontier Sciences, The University of Tokyo, Department of Ocean Floor Geoscience, Atmosphere and Ocean Research Institute, The University of Tokyo, Kashiwanoha, Kashiwa, Japón) y Mike Church (Departamento of Geography, University of British Columbia, Vancouver, Canadá).

\section{REFERENCIAS}

MUNHÁ, 2003. Recognizing possible tsunami sediments. En: The Ultradissipative Environment of the Tagus Estuary (Portugal). Congresso Coastal Sediments' 03, Florida, USA.

\section{ARAOKA, D., M. INOUE, A. SUZUKI, Y. YOKOYAMA, R.L. EDWARDS, H. CHENG, H. MATSUZAKI, H. KAN, N. SHIKAZONO y H. KAWAHATA, 2010. Historic 1771 Meiwa tsunami confirmed by high-resolution U/Th dating of massive Po- rites coral boulders at Ishigaki Island in the Ryukyus, Japan, Geochemical Geophysical Geosystems 11, p. Q06014.}

ATWATER, B.F., 1987. Evidence for great Holocene earthquakes along the outer coast of Washington State. Science 236:942-944.

BAHLBURG, H. y R. WEISS., 2007. Sedimentology of the December 26, 2004, Sumatra tsunami deposits in eastern India (Tamil Nadu) and Kenya. International Journal of Earth Sciences 96:1195-1209.

BONDEVIK, S., J. MANGERUD, S. DAWSON, A. DAWSON y O. LOHNE., 2005. Evidence for three North Sea tsunamis at the Shetland Islands between 8000 and 1500 years ago. Quaternary Science Reviews 24:1757-1775.

BOURGEOIS, J., 2009. Geologic effects and records of tsunamis. En: Robinson, A.R. y E.N. Bernard (eds) The Sea, Volume 15: Tsunamis. Harvard University Press: 5391.

BRYANT, E.A., R.W. YOUNG y D.M. PRICE., 1992. Evidence of tsunami sedimentation on the southeastern coast of Australia. Journal of Geology 100:753-765.

BRYANT, E.A., R.W. YOUNG y D.M. PRICE, 1996. Tsunami as a major control on coastal evolution, southeastern Australia. Journal of Coastal Research 12: 831-840. 
BRYANT, E.A. y S.K. HASLETT., 2002. Was the AD 1607 coastal flooding event in the Severn Estuary and Bristol Channel (UK) due to a tsunami. Archaeology in the Severn Estuary 13:163-167.

BRYANT, E.A. y S.K. HASLETT., 2007. Catastrophic wave erosion, Bristol Channel, United Kingdom: impact of tsunami?. Journal of Geology, 115:253-269.

BRYANT, E.A. y J. NOTT., 2001. Geological indicators of large tsunami in Australia. Natural Hazards 24:231-249.

CANTALAMESSA, G. y C. DI CELMA., 2005. Sedimentary features of tsunami backwash deposits in a shallow marine Miocene setting, Mejillones Peninsula, northern Chile. Sedimentary Geology 178:259-273.

CHOOWONG, M., N. MURAKOSHI, K. HISADA, T. CHAROENTITIRAT, P. CHARUSIRI, S. PHANTUWONGRAJ, $P$. WONGKOK, A. CHOOWONG, R. SUBSAYJUN, V. CHUTAKOSITKANON, K. JANKAEW y P. KANJANAPAYONT., 2008. Flow conditions of the 2004 Indian Ocean tsunami in Thailand, inferred from capping bedforms and sedimentary structures. Terra Nova 20:141-149.

CHURCH, M., 2010. The trajectory of geomorphology. Progress in Physical Geography 34:265-286.

DABRIO, C. y D. POLO., 2005. Registro Estratigráfico de Tsunamis. Enseñanza de las Ciencias de la Tierra.13.1:37-45.

DAWSON, A.G., I.D.L. FOSTER, S. SHI, D.E. SMITH y D. LONG., 1991. The identification of tsunami deposits in coastal sediment sequences. Science of Tsunami Hazards, 9, 1:73-82.

DAWSON, A.G., 1999. Linking tsunami deposits, submarine slides and offshore earthquakes. Quaternary International 60:119126.
DAWSON, A.G. y S. SHI., 2000. Tsunami deposits. Pure and Applied Geophysics, 157:875-897.

DAWSON, A.G. y I. STEWART., 2007. Tsunami deposits in the geological record. Sedimentary Geology 200:166-183.

DOMINEY-HOWES, D., 2007. Geological and historical records of tsunami in Australia. Marine Geology 239(1-2):99-123.

EIPERT, A, 2004. Optically stimulated luminescence (OSL) dating of sand deposited by the 1960 tsunami in south-central Chile. Bachelor of Arts Degree, Carleton College, Northfield, Minnesota.

ENGEL, M., H. BRÜCKNER, V. WENNRICH, A. SCHEFFERS, D. KELLETAT, A. VÖTT, F. SCHÄBITZ, G. DAUT, T. WILLERSHÄUSER y S. MATTHIAS., 2010. Coastal stratigraphies of eastern Bonaire (Netherlands Antilles): New insights into the palaeo-tsunami history of the southern Caribbean. Sedimentary Geology 231,1-2:14-30.

FAGHERAZZI, S. y X. DU., 2008. Tsunamigenic incisions produced by the December 2004 earthquake along the coasts of Thailand, Indonesia and Sri Lanka. Geomorphology 99:120-129.

FELTON, E.A. y K.A.W. CROOK., 2003. Evaluating the impacts of huge waves on rocky shorelines: an essay review of the book Tsunami - The Underrated Hazard. Marine Geology 197:1-12.

FUJINO, S., F. MASUDA, S. TAGOMORI y D. MATSUMOTO., 2006. Structure and depositional processes of a gravelly tsunami deposit in a shallow marine setting: Lower Cretaceous Miyako Group, Japan. Sedimentary Geology 187:127-138. 
FUJINO, S., H. NARUSE, D. MATSUMOTO, T. JARUPONGSAKUL, A. SPHAWAJRUKSAKUL y N. SAKAKURA., 2009. Stratigraphic evidence for pre2004 tsunamis in southwestern Thailand. Marine Geology 262:25-28.

FUJIWARA, O. y T. KAMATAKI., 2007. Identification of tsunami deposits considering the tsunami waveform: an example of subaqueous tsunami deposits in Holocene shallow bay on southern Boso Peninsula, Central Japan. Sedimentary Geology 200:295-313.

GIANFREDA, F., G. MASTRONUZZI y P. SANSÒ., 2001. Impact of historical tsunamis on a sandy coastal barrier: an example from northern Gargano coast, southern Italy. Natural Hazards and Earth System Sciences 1:1-7.

GOFF, J.R., B.G. MCFADGEN y C. CHAGUÉ-GOFF, 2004., Sedimentary differences between the 2002 Easter storm and the 15th Century Okoropunga tsunami, southeastern North Island, New Zealand. Marine Geology 204:235-250.

GOFF, J.R., W.C. DUDLEY, M.J. DEMAINTENON, G. CAIN Y J.P. CONEY., 2006. The largest local tsunami in 20th century Hawaii. Marine Geology 226:65-79.

GOFF, J.R., E. LANE y J. ARNOLD., 2009. The tsunami geomorphology of coastal dunes. Natural Hazards and Earth System Sciences 9:847-854.

GOFF, J.R., S.L. NICHOL y D. KENNEDY., 2010a. Development of a palaeotsunami database for New Zealand. Natural Hazards 54:193-208.

GOFF, J.R., C. CHAGUÉ-GOFF, C. COURTNEY Y D. DOMINEY-HOWES., 2010b. Analysis of the Mahuika comet impact tsunami hypothesis. Marine Geology 271:292-296.
GOTO, K., S.A. CHAVANICH, F. IMAMURA, P. KUNTHASAP, T. MATSUI, K. MINOURA, D. SUGAWARA y H. YANAGISAWA., 2007. Distribution, origin and transport process of boulders deposited by the 2004 Indian Ocean tsunami at Pakarang Cape, Thailand. Sedimentary Geology 202:821-837.

GOTO, K., K. OKADA y F. IMAMURA., 2009. Characteristics and hydrodynamics of boulders transported by storm waves at Kudaka Island, Japan. Marine Geology 262:14-24.

GOTO, K., K. MIYAGI, H. KAWAMATA y F. IMAMURA., 2010a. Discrimination of boulders deposited by tsunamis and storm waves at Ishigaki Island, Japan. Marine Geology 269:34-45.

GOTO, K., T. KAWANA y F. IMAMURA., 2010b. Historical and geological evidence of boulders deposited by tsunamis, southern Ryukyu Islands, Japan. EarthScience Reviews 102:77-99.

HART, D.E. y G.A. KNIGHT., 2009. Geographic information system assessment of tsunami vulnerability on a dune coast. Journal of Coastal Research 25,1:131-141.

HARTLEY, A., J. HOWELL, M.E. MATHER y G. CHONG., 2001. A possible Plio-Pleistocene tsunami deposit, Hornitos, northern Chile. Revista Geológica de Chile 28:117-125.

HASLETT, S.K. y E.A. BRYANT., 2007. Reconnaissance of historic (post-AD 1000) high-energy deposits along the Atlantic coasts of Southwest Brittany, Ireland and Brittany, France. Marine Geology 242:207-220.

HULUGALLE, N.R., R. JAYA, G.C. LUTHER, M. FERIZAL, S. DAUD, YATIMAN, IRHAS, Z.A. YUFNIATI, F. FERIYANTI, TAMRIN y B. HAN., 2009. Physical properties of tsunami-affected soils in Aceh, Indonesia: 21/2 years alter the tsunami. Catena 77:224-231. 
ILAYARAJA, K. y R.R. KRISHNAMURTHY., 2010. Sediment characterisation of the 26 december 2004 Indian ocean tsunami in Andaman Group of Islands, Bay of Bengal, India. Journal of Coastal Conservation 14,3:215-230.

IMAMURA, F., K. GOTO y S. OHKUBO., 2008. A numerical model for the transport of a boulder by tsunami. Journal of Geophysical Research 113,C01008, doi:10.1029/2007JC004170.

JAFFE, B. y G. GELFENBAUM, 2002. Using tsunami deposits to improve assessment of tsunami risk. Solutions to Coastal Disasters ‘02, ASCE:836-847.

JAFFE, B., G. GELFENBAUM, D. RUBIN, R. PETERS, R. ANIMA, M. SWENSSON, D. OLCESE, L. BERNALES, J. GOMEZ y P. RIEGA, 2003. Tsunami deposits: identification and interpretation of tsunami deposits from the June 23, 2001 Perú tsunami. Proceedings of the International Conference on Coastal Sediments '03. 13 pp.

KEATING, B., F. WHELAN y J. BAILEY-BROCK., 2004. Tsunami deposit at Queen's Beach, Oahu, Hawaii - Initial results and wave modelling. Science of Tsunami Hazards 22,1:23-43.

KELLETAT, D. y A. SCHEFFERS., 2004. Bimodal tsunami deposits - a neglected feature in paleo-tsunami research. Coastline Reports 1:1-20.

KELLETAT, D., S.R. SCHEFFERS y A. SCHEFFERS., 2007. Field Signatures of the SE Asian megatsunami along the west coast of Thailand compared to Holocene Paleo-tsunami from the Atlantic region. Pure and Applied Geophysical 164:413431.
KELlETAT, D., 2008. Comments to Dawson, A.G. and Stewart, I. (2007), Tsunami deposits in the geological record. Sedimentary Geology 211:87-91.

KIRCA, V.S.Ö. y M.S. KABDASH, 2006. Bed profile changes due to tsunami effects on near shore coasts: Tuzla case study. Journal of Coastal Research SI 39 (Proceedings of the 8th International Coastal Symposium):1484-1487.

KORTEKAAS, S. y A.G. DAWSON., 2007. Distinguishing tsunami and storm deposits: an example from Martinhal, SW Portugal. Sedimentary Geology 200:208221.

LAGOS, M., 2000. Tsunamis de origen cercano a las costas de Chile. Revista de Geografía Norte Grande 27:93-102.

LAGOS, M. y M. CISTERNAS., 2004. Depósitos de tsunami como indicadores de riesgo: evidencias sedimentarias. Revista Geográfica de Chile Terra Australis 49:329351.

LAGOS, M. y D. GUTIERREZ., 2005. Simulación del tsunami de 1960 en un estuario del Centro-Sur de Chile. Revista de Geografía Norte Grande 33:5-18.

LE ROUX, J.P. y G. VARGAS., 2005. Hydraulic behaviour of tsunami backflows: insights from their modern and ancient deposits. Environmental Geology 49:65-75.

LE ROUX, J.P., S.N. NIELSEN, H. KEMNITZ y A. HENRÍQUEZ., 2008. A Pliocene mega tsunami and associated features in the Ranquil Formation, southern Chile. Sedimentary Geology 203:164-180.

LOWE, D.J. y W.P. DE LANGE., 2000. Volcano-meteorological tsunamis, the c.AD200 Taupo eruption (New Zealand) and the possibility of a global tsunami. Holocene 10,3:401-407. 
LUQUE, L., J. LARIO, C. ZAZO, J.L. GOY, C.J. DABRIO y P.G. SILVA., 2001. Tsunami deposits as paleoseismic indicators: examples from the Spanish coast. Acta Geológica Hispánica 36,3-4:197-211.

LUQUE, L., 2008._El impacto de eventos catastróficos costeros en el litoral del Golfo de Cádiz. Revista Atlántica-Mediterránea de Prehistoria y Arqueología Social 10:131153.

MAOUCHE, S., C. MORHANGE y M. MEGHRAOUI., 2009. Large boulder accumulation on the Algerian coast evidence tsunami events in the western Mediterranean. Marine Geology 262:96-104.

MASTRONUZZI, G. y P. SANSÒ., 2004. Large boulder accumulations by extreme waves along the Adriatic coast of southern Apulia (Italy). Quaternary International 120:173-184.

MASTRONUZZI, G., C. PIGNATELli, P. SANSÒ y G. SELLERI., 2007. Boulder accumulations produced by the 20th February 1743 tsunami along the coast of southeastern Salento (Apulia region, Italy). Marine Geology 242,1-3:191-205.

MATSUMOTO, T., M. KIMURA, M. NAKAMURA y T. ONO., 2001. Largescale slope failure and active erosion occurring in the southwestern Ryukyu fore-arc area, Natural Hazards and Earth System Sciences 1:203-211.

MCFADGEN, B.G. y J.R. GOFF., 2007. Tsunamis in the New Zealand archaeological records. Sedimentary Geology 200:263-274.

MCMURTRY, G., P. WATTS, G. FRYER, J.R. SMITH y F. IMAMURA., 2004. Giant landslides, mega-tsunamis and paleo-sea level in the Hawaiian islands. Marine Geology 203:219-233.
MCMURTRY, G.M., D.R. TAPPIN, P.N. SEDWICK, I. WILKINSON, J. FIETZKE y B. SELLWOOD., 2007. Elevated marine deposits in Bermuda record a late Quaternary Megatsunami. Sedimentary Geology 200,3-4:155-165.

MHAMMDI, N., F. MEDINA, D. KELLETAT, M. AHMAMOU y L. ALOUSSI., 2008. Large boulders along the Rabat coast (Morocco); possible emplacement by the November, 1st, 1755 A.D. tsunami. Science of Tsunami Hazard, Honolulu, 27,1:17-30.

MINOURA, K., F. IMAMURA, D. SUGAWARA, Y. KONO y T. IWASHITA., 2001. The 869 Jogan tsunami deposit and recurrence interval of large-scale tsunami on the Pacific coast of northeast Japan. Journal of Natural Disaster Science, 23, pp. 83-88.

MITCHELL, A.J., P.A. ALLISON, M.D. PIGGOTT, G.J._GORMAN, CH.C. PAIN y G.J. HAMPSON., 2010. Numerical modelling of tsunami propagation with implications for sedimentation in ancient epicontinental seas: the Lower Jurassic Laurasian Seaway. Sedimentary Geology 228,3-4:8197.

MOORE, A. y B. JAFFE, 2007. Geologic evidence of tsunamis. En: GONZALEZ, F.I., E. BERNARD, P. DUNBAR, E. GEIST, B. JAFFE, U. KANOGLU, J. LOCAT, H. MOFJELD, A. MOORE, C. SYNOLAKIS, V. TITOV y R. WEISS (eds). Scientific and Technical Issues in Tsunami Hazard Assessment of Nuclear Power Plant Sites. NOAA Tech. Memo. OAR PMEL-136, Pacific Marine Environmental Laboratory, Seattle, Washington.

MORTON, R.A., G. GELFENBAUM y B.E. JAFFE., 2007. Physical criteria for distinguishing sandy tsunami and stormdeposits using modern examples. Sedimentary Geology 200:184-207. 
NANAYAMA，F., R. FURUKAWA，K. SHIGENO, A. MAKINO, Y. SOEDA y Y. IGARASHI., 2007. Nine unusually large tsunami deposits from the past 4000 years at Kiritappu march along the southern Kuril trench. Sedimentary Geology 200:275-294.

NARAYAN, J.P., M.L. SHARMA y B.K. MAHESHWARI., 2005. Effects of medu and coastal topography on the damage pattern during the recent Indian Ocean tsunami along the coast of Tamil Nadu. Science of Tsunami Hazards 23,2:9-18.

NICHOL, S.L., C. CHAGUÉ-GOFF, J.R. GOFF, M. HORROCKS, B.G. MCFADGEN y L.C. STROTZ., 2010. Geomorphology and accommodation space as limiting factors on tsunami deposition: Chatham Island, southwest Pacific Ocean. Sedimentary Geology 229,1-2,:41-52.

NISHIMURA, Y. y N. MIYAJI., 1995. Tsunami deposits from the 1993 Southwest Hokkaido earthquake and the 1640 Hokkaido Komagatake eruption, Northern Japan. Pure and Applied Geophysics 144,3-4:719733.

NISHIMURA, Y., M. NAKAGAWA, J. KUDUON y J. WUKAWA., 2005. Timing and scale of tsunamis caused by the 1994 Rabaul eruption, east New Britain, Papua New Guinea. Advances in Natural and Technological Hazards Research 23,I:43-56.

NOVOA, J.E, 1995. Potencial tsunami effects in La Serena area North-Central Chile. Late Quaternary Coastal Record of Rapid Change: Application to Present and Future Conditions. Antofagasta. Chile. 16 - 26 November 1995 (IGCP Project 367).

NOTT, J., 2000. Records of prehistoric tsunamis from boulder deposits evidence from Australia. Science of Tsunami Hazards 18:3-14.
NOTT, J.F., 2003. Waves, coastal boulders and the importance of the pre-transport setting. Earth and Planetary Science Letters 210:269276.

NOTT, J.F., 2004. The tsunami hypothesis comparisons of the field evidence against the effects, on the Western Australian coast, of some of the most powerful storms on Earth. Marine Geology 208:1-12.

OLIVEIRA, M.A., C. ANDRADE, M.C. FREITAS y P.J. COSTA., 2009. Modelling volume transfer between beach-foredune and the backshore at Boca do Rio lowland, Algarve (Portugal) by the 1755 Lisbon tsunami. Journal of Coastal Research SI56 (Proceedings of the 10th International Coastal Symposium):1547-1551.

PARIS, R., P. WASSMER, J. SARTOHADI, F. LAVIGNE, B. BARTHOMEUF, E. DESGAGES, D. GRANCHER, P. BAUMERT, F. VAUTIER, D. BRUNSTEIN y C. GOMEZ., 2009. Tsunamis as geomorphic crisis: lessons from the December 26, 2004 tsunami in Lhok Nga, west Banda Aceh (Sumatra, Indonesia). Geomorphology 104:59-72.

PARIS, R., J. FOURNIER, E. POIZOT, S. ETIENNE, J. MORIN, F. LAVIGNE y P. WASSMER., 2010. Boulder and fine sediment transport and deposition by the 2004 tsunami in Lhok Nga (western Banda Aceh, Sumatra, Indonesia): a coupled offshore-onshore model. Marine Geology 268:43-54.

PASKOFF, R., 1991. Likely occurrence of a mega-tsunami in the middle Pleistocene near Coquimbo, Chile. Revista Geológica de Chile 18:87-91.

PÉREZ-TORRADO,F.J., R.PARIS, M.C. CABRERA，J.C. CARRACEDO, J.L. SCHNEIDER, P. WASSMER, H. GUILLOU y D. GIMENO., 2002. Depósitos de tsunami en el valle de Agaete, Gran Canaria (Islas Canarias). Geogaceta 32:75-78. 
PETERS, R., B. JAFFE y G. GELFENBAUM., 2007. Distribution and sedimentary characteristics of tsunami deposits along the Cascadia margin of western North America. Sedimentary Geology 200:372-386.

PIGNATELLI, C., P. SANS y G. MASTRONUZZI., 2009. Evaluation of tsunami flooding using geomorphological evidence. Marine Geology 260:6-18.

PINEGINA, T. K. y J. BOURGEOIS., 2001. Historical and paleo-tsunami deposits on Kamchatka, Russia; long-term chronologies and long-distance correlations. Natural Hazards and Earth System Sciences 1:177185.

PUGA-BERNABÉU, A., J.C. BRAGA y J.M. MARTÍN., 2007. High-frequency cycles in Upper-Miocene ramp-temperate carbonates (Sorbas Basin, SE Spain). Facies 53:329-345.

RICHMOND, B., B.E. JAFFE, G. GELFENBAUMUM y R.A. MORTON, 2006. Geologic impacts of the 2004 Indian Ocean tsunami on Indonesia, Sri Lanka, and the Maldives. En: SCHEFFERS, A. y D. KELLETAT (eds.) Tsunamis, Hurricanes and Neotectonics as Driving Mechanisms in Coastal Evolution. Zeitschrift für Geomorphologie N.F., Suppl. 146:235-251.

RUEGG, A. y J.C. RUDLOFF., 2009. Interseismic strain accumulation measured by GPS in the seismic gap between Constitución and Concepción in Chile. Physics of the Earth and Planetary Interiors 175:78-85.

SANTOS, A.S., S. KOSHIMURA y F. IMAMURA., 2009. The Lisbon Tsunami: tsunami source determination and its validation. Journal of Disaster Research 4:4152.

SCHEFFERS, A.M., 2002. Palaeotsunami evidence from boulder deposits on Curacao and Bonaire. Science of Tsunami Hazards 20:26-38.
SCHEFFERS, A. y D. KELLETAT., 2003. Sedimentologic and geomorphologic tsunami imprints worldwide: a review. Earth Science Reviews 63,1-2:83-92.

SCHEFFERS, A., 2004. Tsunami imprints on the Leeward Netherlands Antilles (Aruba, Curacao, Bonaire) and their relation to other coastal problems. Quaternary International 120:163-172.

SCHEFFERS, A. y D. KELLETAT, 2004. Bimodal tsunami deposits - a neglected feature in paleotsunami research. En: SCHERNEWSKI, G. y T. DOLCH (eds) Geographie der Meere und Küsten. Coastline Reports 1:67-75.

SCHEFFERS, A. y S. SCHEFFERS., 2007. Tsunami deposits on the coastline of west Crete (Greece). Earth and Planetary Science Letters 259:613-624.

SCHEFFERS, S., A. SCHEFFERS, D. KELLETAT y E.A. BRYANT., 2008. The Holocene paleo-tsunami history of West Australia. Earth and Planetary Science Letters 270:137-146.

SINGARASUBRAMANIAN S.R., M.V. MUKESH, K. MANOHARAN, S. MURUGAN, D. BAKKIARAJ, A. JOHN PETER y P. SERALATHAN., 2006. Sediment characteristics of the M-9 tsunami event between Rameswaram and Thoothukudi, Gulf of Mannar, southeast coast of India; Science of Tsunami Hazards 25:160-172.

SPISKE, M., Z. BÖRÖCZ y H. BAHLBURG., 2008. The role of porosity in discriminating between tsunami and hurricane emplacement of boulders. A case study from the Lesser Antilles, southern Caribbean. Earth and Planetary Science Letters 268:384-396. 
SRINIVASALU, S., N. RAJESHWARA RAO, N. THANGADURAI, M.P. JONATHAN, P.D. ROY, V. RAM MOHAN Y P. SARAVANAN., 2009. Characteristics of 2004 tsunami deposits of the northern Tamil Nadu coast, southeastern India. Boletín de la Sociedad Geológica Mexicana 61,1:111118.

SRISUTAM, C. y J.F. WAGNER., 2010. Reconstructing tsunami run-up from the characteristics of tsunami deposits on the Thai Andaman Coast. Coastal Engineering 57:493-499.

SUGAWARA, D., K. MINOURA, N. NEMOTO, SH. TSUKAWAKI, K. GOTO y F. IMAMURA., 2009. Foraminiferal evidence of submarine sediment transport and deposition by backwash during the 2004 Indian Ocean tsunami. The Island Arc 18,3:513-525.

SUZUKI, A., Y. YOKOYAMA, H. KAN, K. MINOSHIMA, H. MATSUZAKI, N. HAMANAKA y H. KAWAHATA., 2008. Identification of 1771 Meiwa Tsunami deposits using a combination of radiocarbon dating and oxygen isotope microprofiling of emerged massive Porites boulders. Quaternary Geochronology 3:226-234.

SYNOLAKIS, C.E., P. LIU, H. YEH y G. CARRIER., 1997. Tsunamigenic seafloor deformations. Science 278:598-600.

SZCZUCIŃSKI, W., N. CHAIMANEE, P. NIEDZIELSKI, G. RACHLEWICZ, D. SAISUTTICHAI, T. TEPSUWAN, S. LORENC y J. SIEPAK., 2006. Environmental and geological impacts of the 26 December 2004 tsunami in coastal zone of Thailand - overview of short and long-term effects. Polish Journal of Environmental Studies 15(5):793-810.
VÖTT, A., H. BRÜCKNER, M. MAY, F. LANG y S. BROCKMÜLLER., 2007. Late Holocene tsunami imprint at the entrance of the Ambrakian Gulf (NW Greece). Mediterranée 108:43-57.

WEBSTER, J.M., D.A. CLAGUE y J.C. BRAGA., 2007. Support for the giant wave hypothesis: evidence from submerged terraces off Lanai, Hawaii. International Journal of Earth Sciences 96:517-524.

WHELAN, F. y D. KELLETAT., 2002. Geomorphologic evidence and relative and absolute dating results of tsunami events on Cyprus. Science of Tsunami Hazards 20,1:3-18.

WILLIAMS, D.M. y A.M. HALL., 2004. Cliff-top megaclast deposit of Ireland, a record of extreme waves in the North Atlantic: storms or tsunamis?. Marine Geology 206:101-117.

YAMANO, H., O. ABE, E. MATSUMOTO, H. KAYANNE, N. YONEKURA y P. BLANCHON., 2003. Influence of wave energy on Holocene coral reef developments: an example from Ishigaki Island, Ryukyu Islands, Japan. Sedimentary Geology

VÖTT, A., H. BRÜCKNER, M. MAY, F. LANG y S. BROCKMÜLLER. 2007. Late Holocene tsunami imprint at the entrance of the Ambrakian Gulf (NW Greece). Mediterranée 108:43-57.

WEBSTER, J.M., D.A. CLAGUE y J.C. BRAGA, 2007. Support for the giant wave hypothesis: evidence from submerged terraces off Lanai, Hawaii. International Journal of Earth Sciences 96:517-524.

WHELAN, F. y D. KELLETAT, 2002. Geomorphologic evidence and relative and absolute dating results of tsunami events on Cyprus. Science of Tsunami Hazards 20,1:3-18. 
WILLIAMS, D.M. y A.M. HALL, 2004. Cliff-top megaclast deposit of Ireland, a record of extreme waves in the North Atlantic: storms or tsunamis?. Marine Geology 206:101-117.
YAMANO, H., O. ABE, E. MATSUMOTO, H. KAYANNE, N. YONEKURA y P. BLANCHON, 2003. Influence of wave energy on Holocene coral reef developments: an example from Ishigaki Island, Ryukyu Islands, Japan. Sedimentary Geology 159:2741.

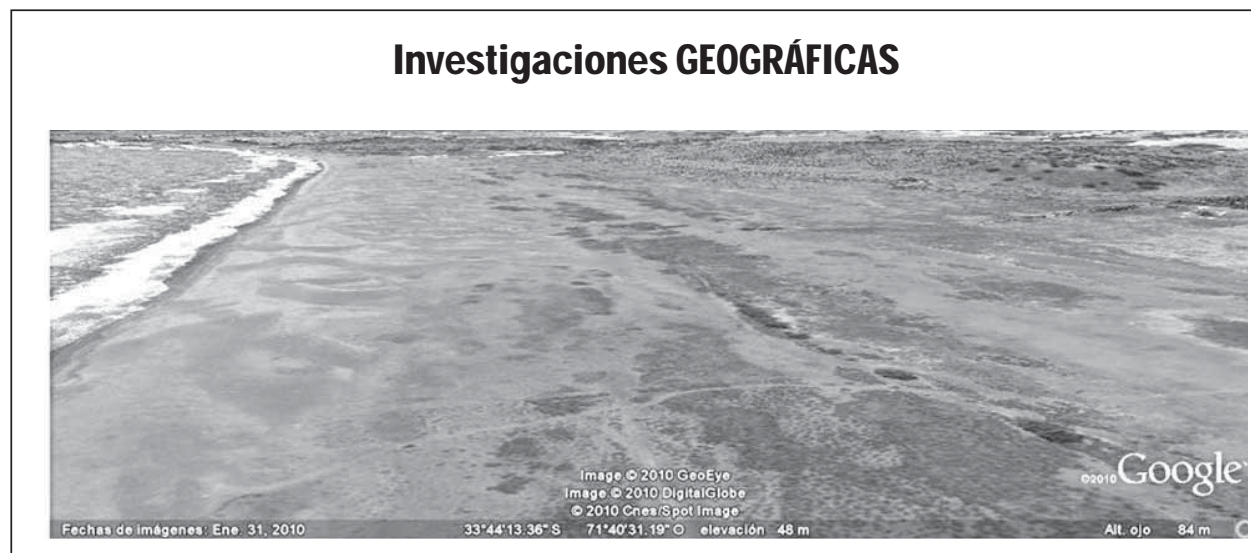

Litoral del Humedal El Yali, Chile Central (3344’56”S-7142’51”W): Cinturón barjánico de barjanoides sobre cordón de playa presente existente desde más de 55 años. Estado al 31.01.10, pre-tsunami 2010.

Shoreline of El Yali wetland: Barchan belt of barchanoids overlaying the present beach ridge since 55 year or more. State by 01.31.10. (Image Google Earth scene in $3 D$ by J.F.Araya-Vergara).

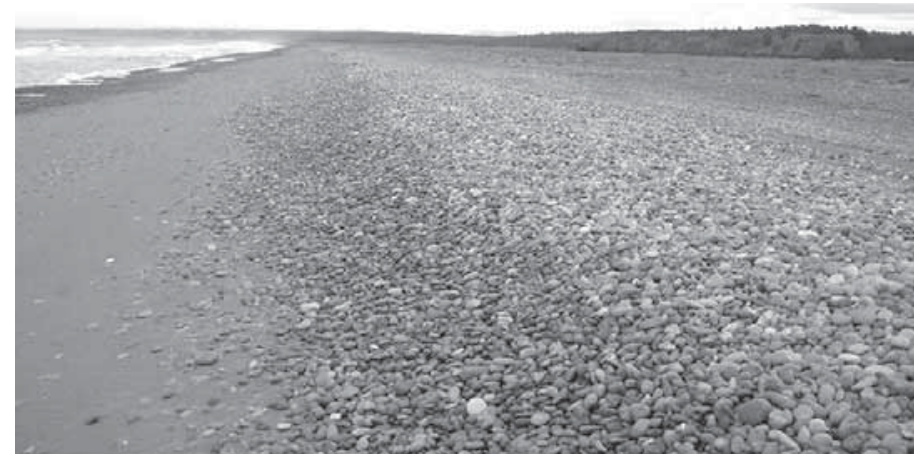

Litoral del Humedal El Yali, Chile Central (3344’56”S-7142’51”W): Cinturón de barjanoides removido por vaivén tsunámico del 27.02.10. Run-out 550 m y run-up 7 m. Paisaje residual: playa de gravas mixta-bisecuencial. Arenas dunares diseminadas por vaivén enmascaran un cordón de playa holocénico.

Shoreline of El Yali wetland: Barchanoid belt removed by 27.02.10 tsunamic swash. Run-out $550 \mathrm{~m}$ and run-up $\sim 7 \mathrm{~m}$. Residual landscape: Mixed-two sequences gravel beach. Sands sparsed by swash mask a Holocenic beach ridge. (Photo J.F. Araya Vergara, 11.12.10, (C2010 Invest. Geogr. Chile, al rights reserved) 